\title{
Being Bad in a Video Game Can Make Us Morally Sensitive
}

\author{
Matthew Grizzard, PhD, Ron Tamborini, PhD, ${ }^{2}$ Robert J. Lewis, $\mathrm{PhD},{ }^{3}$ Lu Wang, $\mathrm{MA},{ }^{2}$ and Sujay Prabhu, $\mathrm{MA}^{2}$
}

\begin{abstract}
Several researchers have demonstrated that the virtual behaviors committed in a video game can elicit feelings of guilt. Researchers have proposed that such guilt could have prosocial consequences. However, this proposition has not been supported with empirical evidence. The current study examined this issue in a $2 \times 2$ (video game play vs. real world recollection $\times$ guilt vs. control) experiment. Participants were first randomly assigned to either play a video game or complete a memory recall task. Next, participants were randomly assigned to either a guilt-inducing condition (game play as a terrorist/recall of acts that induce guilt) or a control condition (game play as a UN soldier/recall of acts that do not induce guilt). Results of the study indicate several important findings. First, the current results replicate previous research indicating that immoral virtual behaviors are capable of eliciting guilt. Second, and more importantly, the guilt elicited by game play led to intuition-specific increases in the salience of violated moral foundations. These findings indicate that committing "immoral" virtual behaviors in a video game can lead to increased moral sensitivity of the player. The potential prosocial benefits of these findings are discussed.
\end{abstract}

\section{Introduction}

$\mathbf{S}$ EVERAL RECENT STUDIES HAVE DEMONSTRATED that committing immoral behaviors in a video game can elicit guilt. ${ }^{1,2}$ Guilt is a moral emotion that provides "immediate and salient feedback on our social and moral acceptability. When we sin, transgress or err, aversive feelings of shame, guilt, or embarrassment are likely to ensue.",3(p347) Because moral emotions are anticipatory as well as consequential (i.e., one can anticipate feeling guilty before one commits a transgression), ${ }^{3}$ researchers ${ }^{1,2}$ have argued that committing immoral actions in video games may lead to prosocial effects. If a player "feels guilty for a certain behavior or choice, a certain level of conscious consideration of the repercussion of one's behavior is implied. Games could provide an important outlet for not only making moral decisions, but also reflecting upon (and perhaps mentally rehearing) what the right choices and behaviors are."1(p614) The current study seeks to test whether playing an immoral role in a video game can lead to increased sensitivity of relevant moral intuitions through the elicitation of guilt.

Tamborini $^{4,5}$ has recently proposed a model of intuitive morality and exemplars (MIME) that combines current advances in moral psychology with media effects theories to explain the influence of mediated experiences on individuals' moral judgments. Moral foundations theory (MFT) ${ }^{6}$ provides the theoretical foundation for the MIME's conceptualization of morality. MFT proposes that human morality is the result of five evolutionarily derived intuitions: care (related to empathy and violence), fairness (related to justice considerations), loyalty (related to ingroup biases), authority (related to respect for dominance hierarchies), and purity (related to sanctity and avoidance of bodily contamination). Tamborini argues that these moral intuitions drive individuals' evaluations of media, such as video games. ${ }^{4,5}$ Previous research on video game play demonstrates that the salience of these moral intuitions can drive decision making in video games. ${ }^{1,7}$ Notably, research by Weaver and Lewis did not find a significant correlation between sensitivity of these moral intuitions and guilt experienced after game play. ${ }^{1}$ However, this research examined moral intuition salience prior to game play, and not the ability of game play to increase intuition salience.

The MIME also predicts that mediated experiences can increase the salience of content-relevant intuitions. ${ }^{4,5}$ Applied to video game play and, more specifically, the game utilized in the current study, engaging in unjustified violence in a video game should increase the salience of fairness and

\footnotetext{
${ }^{1}$ Department of Communication, University at Buffalo, The State University of New York, Buffalo, New York.

${ }^{2}$ Department of Communication, Michigan State University, East Lansing, Michigan.

${ }^{3}$ Department of Advertising and Public Relations, University of Texas at Austin, Austin, Texas.
} 
care intuitions (i.e., the intuitions violated by engaging in unjust violence). Related research has shown that exposure to television programming can increase the salience of content-relevant intuitions, ${ }^{8,9}$ but the observed effects have been somewhat weak. This weakness may simply indicate that media's ability to affect these intuitions is weak. However, other explanations are also possible. One explanation might be that media's influence on these moral intuitions is mediated by emotional responses not measured in earlier research, such as guilt.

Previous investigations argue that video games offer moral agency to players that is absent in narrative media such as televised drama and news. In narrative media, viewers simply watch moral decisions being made by others, but in video games, players often make the decision to be moral (or immoral). ${ }^{1}$ The present study began by considering situations where people engaged in (or recalled) immoral or moral behaviors that vary in their ability to induce guilt. It then built on the expectations that (a) the moral agency afforded by video game play will prompt immoral game play behavior to have an influence on moral intuition salience, and (b) moral emotions such as guilt will serve as a mediator for the influence of video game play on moral intuition salience. As such, the current study seeks to test the following hypotheses:

\section{H1: Engaging in immoral behavior in a video game will lead to higher levels of guilt than engaging in moral behavior.}

H2: Guilt experienced from video game play will lead to increased salience of the content-relevant intuitions of care and fairness.

H3: Guilt mediates the relationship between engaging in immoral behavior and increases in content-relevant intuitions.

With regard to $\mathrm{H} 2$, the video game selected for use in the study is expected to produce an increase in the salience of the care and fairness intuitions specifically because of committing unjustified violence in the game (i.e., a simultaneous violation of the care and fairness intuitions). To test for the potential that guilt can selectively increase the salience of the specific moral intuitions whose violation yielded the guilt, we chose to elicit guilt utilizing two procedures: video game play and memory recall. ${ }^{\mathrm{a}}$ In the current study, we should expect both guilt inductions to elicit guilt. However, we should only expect game-elicited guilt to correlate with the content-relevant intuitions of care and fairness, as the guilt elicited by the game should be associated systematically and exclusively with these two moral intuitions. The guilt elicited by an undirected recall of memories, on the other hand, should not be systematically related to any intuition. That is, recalled guilt should vary randomly across all intuitions, as participants should be equally likely to recall guilt associated with a violation of the care and fairness intuitions as they are to recall guilt associated with the other three intuitions. This procedure can provide both convergent and discriminant evidence. As such, we expect a positive relationship between guilt and the salience of the care and fairness intuitions for participants in the video game conditions (convergent evidence). Guilt and intuition salience should be unrelated for all other intuitions in both the video game and memory recall conditions (discriminant evidence).

\section{Method}

\section{Participants}

Participants $(N=185)$ were recruited from classes at a large, Midwestern university in the United States, and received course credit for their participation. Participants' ages ranged from 18 to 29 years $(M=20.18$ years, $S D=1.70,122$ females).

\section{Design}

A $2 \times 2$ (medium: video game play vs. memory recall $\times$ condition: guilt vs. control) experiment was conducted to test the hypotheses. Participants were first randomly assigned to either play a video game or complete a memory recall task, and then randomly assigned to a guilt or control condition.

\section{Materials}

Participants in the video game conditions played a modified version of a first-person shooter game utilized in previous research. ${ }^{2}$ Video game play participants in the guilt condition played as a terrorist soldier, while participants in the control condition played as a UN soldier. The game itself informed participants of their character's motivations to ensure that the experimenter did not bias results. Participants in the memory recall conditions were asked to remember and describe in a short paragraph a time in which they felt particularly guilty (the guilt condition) or an ordinary day (the control condition). Game play lasted for approximately 10 minutes; the memory recall procedure was not timed, but participants wrote on average 51.79 words $(S D=22.99)$.

\section{Measures}

After completing the video game or the memory recall, participants completed a 3-item guilt scale from prior studies: " "To what extent do you feel..." "regret," "sorry about something you've done," and "like you've done something wrong." Next, participants completed the 30-item moral foundations questionnaire (MFQ), designed to assess the salience of the five moral intuitions. ${ }^{10}$ At the end of the study, participants in the video game conditions completed a manipulation check asking them whether they played as a terrorist or a UN soldier. Participants $\left(n_{\text {Terrorist }}=15, n_{\mathrm{UN}}\right.$ Soldier $\left.=15\right)$ who answered incorrectly or could not remember what type of character they controlled were excluded from further analysis, as these responses indicate a failure of the primary manipulation.

After obtaining a valid and reliable factor loading through confirmatory factor analyses, composites were created for the guilt scale and the five moral intuition scales. The reliabilities were guilt $\alpha=0.88$, care $\alpha=0.60$, fairness $\alpha=0.66$, loyalty $\alpha=0.61$, authority $\alpha=0.59$, and purity $\alpha=0.72$. Although some of these reliabilities may appear less than optimal, they are consistent with previous uses of the MFQ. ${ }^{11}$

\section{Results}

Prior to hypotheses testing, a zero-order correlation matrix was created, as well as separate correlation matrices for the video game conditions and the memory recall conditions (see Table 1). The significant positive correlation between guilt 
Table 1. Correlations Among Study Variables

\begin{tabular}{|c|c|c|c|c|c|c|}
\hline & Condition & Guilt & Care & Fairness & Loyalty & Authority \\
\hline \multicolumn{7}{|c|}{ Zero-order correlations $(N=155)$} \\
\hline Guilt & $0.36 * *$ & 1 & & & & \\
\hline Care & 0.07 & $0.19 *$ & 1 & & & \\
\hline Fairness & 0.05 & 0.10 & $0.54 * *$ & 1 & & \\
\hline Loyalty & -0.09 & -0.02 & $0.31 * *$ & 0.07 & 1 & \\
\hline Authority & 0.00 & 0.07 & $0.38 * *$ & $0.40 * *$ & $0.48 * *$ & 1 \\
\hline Purity & 0.06 & 0.05 & $0.35 * *$ & $0.30 * *$ & $0.50 * *$ & $0.67 * *$ \\
\hline \multicolumn{7}{|c|}{ Video game play conditions $(n=68)$} \\
\hline Guilt & $0.25^{*}$ & 1 & & & & \\
\hline Care & 0.07 & $0.28 *$ & 1 & & & \\
\hline Fairness & 0.13 & $0.35 * *$ & $0.73 * *$ & 1 & & \\
\hline Loyalty & -0.07 & 0.00 & $0.36 * *$ & 0.06 & 1 & \\
\hline Authority & 0.11 & 0.19 & $0.53 * *$ & $0.48 * *$ & $0.46^{* *}$ & 1 \\
\hline Purity & 0.17 & 0.14 & $0.49 * *$ & $0.40 * *$ & $0.52 * *$ & $0.59 * *$ \\
\hline \multicolumn{7}{|c|}{ Memory recall conditions $(n=87)$} \\
\hline Guilt & $0.49^{*}$ & 1 & & & & \\
\hline Care & 0.07 & 0.06 & 1 & & & \\
\hline Fairness & 0.02 & -0.11 & $0.35 * *$ & 1 & & \\
\hline Loyalty & 0.12 & -0.03 & $0.24 *$ & 0.07 & 1 & \\
\hline Authority & 0.08 & 0.01 & $0.25^{*}$ & $0.32 * *$ & $0.50 * *$ & 1 \\
\hline Purity & 0.02 & -0.02 & $0.24 *$ & $0.21 *$ & $0.50 * *$ & $0.72 * *$ \\
\hline
\end{tabular}

Note: Condition is dummy coded as $1=$ guilt condition, $0=$ control condition.

$* p<0.05 ; * * p<0.01$

and condition for the video game conditions $(r=0.25$, $p<0.05)$ is consistent with Hypothesis 1 , indicating that immoral video game behavior leads to feelings of guilt. Replicating prior research, ${ }^{1,2}$ participants playing as terrorist soldiers $(M=4.55, S D=2.76)$ felt significantly guiltier than participants playing as UN soldiers $(M=3.24, S D=2.41)$, $t(66)=2.08, p=0.04$, Cohen's $d=0.51$.

With regard to the memory recall conditions, the significant correlation between guilt and condition $(r=0.49, p<$ $0.01)$ provides evidence that the memory recall group recounting an experience in which they felt guilty $(M=5.79$, $S D=1.89$ ) experienced significantly more guilt than the participants recounting an ordinary day $(M=3.75, S D=1.78)$, $t(85)=5.19, p<0.001$, Cohen's $d=1.13$.

The correlation matrices (see Table 1) are consistent with the expected patterns suggested by Hypothesis 2. For participants in the video game conditions, guilt is significantly positively correlated with the salience of the care $(r=0.28$, $p=0.02)$ and fairness $(r=0.35, p<0.003)$ intuitions (i.e., the intuitions violated in game play), but not significantly correlated with the loyalty $(r=0.00, p=1.00)$ authority $(r=0.19$, $p=0.12)$ or purity $(r=0.14, p=0.25)$ intuitions. In addition, for participants in the memory recall conditions, guilt does not significantly correlate with the salience of any intuitions. This specific pattern of significant correlations is consistent with Hypothesis 2, indicating that the guilt elicited by game play should lead to increases in the content-relevant intuitions of care and fairness and only those intuitions.

To provide further evidence of the intuition-specific effects of guilt, structural equation models were examined to determine whether guilt mediated the increase in the salience of content-relevant intuitions. Due to the high average correlation between the intuitions (mean $r=0.40$ ) and the potential for multicollinearity to distort results and substantive conclusions, ${ }^{12} 10$ separate structural equation models were conducted (one for each of the five intuitions separated by the two media). Criteria for evaluating the structural equation models were established a priori, and consisted of (a) significant paths between variables, (b) $\mathrm{CMIN} / d f<2.00$, (c) a comparative fit index $(\mathrm{CFI})>0.95$, (d) the root mean square residual of approximation (RMSEA) $<0.06$, and (e) the standardized root mean square residual $(\mathrm{SRMR})<0.08$. $^{13}$ The basic outline of the structural equation models tested can be seen in Figure 1, and the results of these tests are shown in

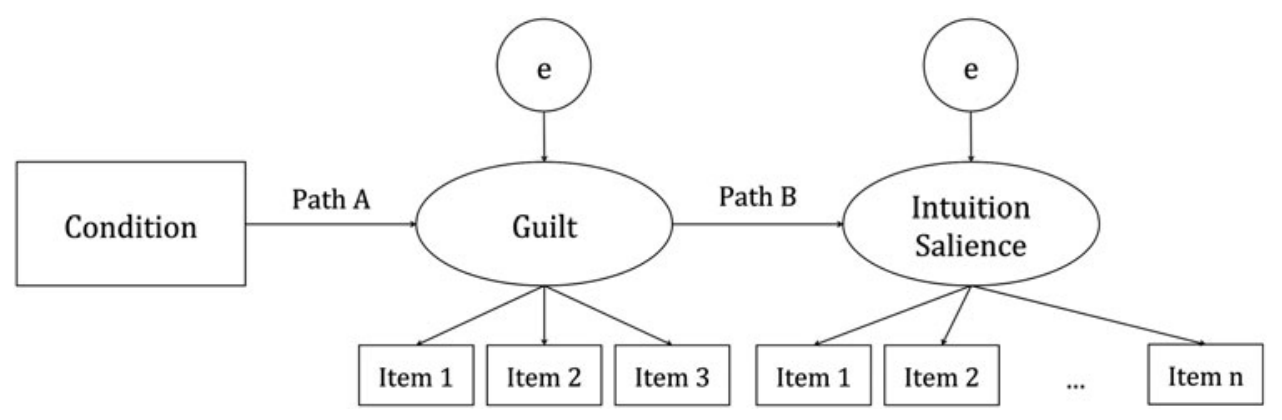

FIG. 1. Basic outline of the structural equation models designed to test the mediating role of guilt. 
Table 2. Results of the structural equation models are consistent with Hypothesis 3 . In the current study, guilt acted as a mediator between video game play and intuition salience for the care and fairness intuitions only.

\section{Discussion}

Results of the current study suggest a link between the performance of antisocial behaviors in video games and the potential for prosocial effects. ${ }^{1,2}$ These findings indicate that committing "immoral" virtual behaviors in a video game can increase the salience of content-relevant intuitions. Notably, this increase means that instead of becoming less sensitive to moral violations, players who commit moral transgression in video games actually become more sensitive to moral violations, if they feel guilt. Importantly, only models examining an increase in the salience of moral intuitions violated in game play (i.e., care and fairness) fit the data. All other models fail to meet the a priori determined criteria. These results provide strong convergent and discriminant validity for the potential that playing an immoral character in a video game leads to an increased salience of content-relevant intuitions mediated by the moral emotion of guilt. Furthermore, by utilizing random assignment and a sequential model, the findings provide evidence of causation.

The increase in the salience of content-relevant intuitions observed in the current study is particularly telling when combined with the results of prior work. In an earlier study, Weaver and Lewis examined whether the salience of the moral intuitions prior to game play correlated with guilt. ${ }^{1}$ Their work found no significant correlation between moral intuition salience prior to game play and guilt experienced from video game play, with a 0.02 correlation between guilt and care salience and a 0.10 correlation between guilt and fairness salience. The significant positive correlations of 0.28 for the guilt-care correlation and 0.35 for the guiltfairness correlation observed here strongly contrast with their prior work, suggesting that the correlations observed here indicate the ability of game play to increase the salience of the intuitions rather than a natural correlation. In addition, the differentiation of these correlations between the video game and memory recall conditions reinforces the potential that increases in intuition salience are related exclusively to guilt-eliciting stimuli. In other words, guilt does not function in a general manner that leads to an increase in all intuitions; guilt only affects the salience of violated intuitions.

The implications for these findings are important practically and theoretically. The findings here demonstrate the potential for emotional experiences that result from media exposure to alter the intuitive foundations upon which humans make moral judgments. This is particularly relevant for video game play, where habitual engagement with the media is the norm for a small but considerably important group of media users. $^{14}$

Overall, the findings suggest two possibilities. First, repeated play as an immoral character may repeatedly activate guilt and its resultant influence on the increased importance of care and fairness. Under these conditions, we might expect that repeated play as an immoral character would lead gamers to become more sensitive to fairness and more caring overall. Alternatively, guilt resulting from playing as an immoral character may habituate from repeated exposures. Under these conditions, we might expect that repeated play would not lead a gamer to become more sensitive to fairness or become more caring overall, especially if the ability of the game to elicit guilt dissipates with repeated play.

A less central finding of the current study indicates that recalling a past real world transgression elicits more guilt than a virtual transgression, as indicated by the larger effect size for the memory recall conditions compared to the video game conditions. This was not wholly unexpected, as the strength of an emotional experience correlates with the probability of that experience to be stored in long-term memory. Accordingly, particularly strong emotional experiences are perhaps the most likely to be recalled. ${ }^{15}$ Despite the difference in effect sizes, the fact that engaging in virtual transgressions was capable of eliciting similar increases in guilt along the same measure as compared to recalling a past transgression indicates that the guilt experienced from game play is functionally similar to real world guilt. As such, the current study provides empirical evidence of a proposition that has been merely assumed in prior research.

Table 2. Numerical Results from the Structural Equation Models Testing Hypothesis 3

\begin{tabular}{|c|c|c|c|c|c|c|}
\hline Intuition & Path A & Path B & $C M I N / d f$ & $C F I$ & RMSEA & $S R M R$ \\
\hline \multicolumn{7}{|c|}{ Video game play conditions } \\
\hline Care & $0.24 *$ & $0.32 * *$ & 0.93 & 1.00 & 0.00 & 0.04 \\
\hline Fairness & $0.24 * *$ & $0.40 * *$ & 0.79 & 1.00 & 0.00 & 0.04 \\
\hline Loyalty & $0.24 * *$ & -0.03 & 1.99 & 0.90 & 0.12 & 0.11 \\
\hline Authority & $0.24 * *$ & 0.26 & 0.92 & 1.00 & 0.00 & 0.05 \\
\hline Purity & $0.24 * *$ & 0.17 & 2.02 & 0.92 & 0.12 & 0.12 \\
\hline \multicolumn{7}{|c|}{ Memory recall conditions } \\
\hline Care & $0.54 * *$ & 0.04 & 1.33 & 0.95 & 0.06 & 0.07 \\
\hline Fairness & $0.54 * *$ & -0.13 & 1.48 & 0.95 & 0.08 & 0.06 \\
\hline Loyalty & $0.54 * *$ & -0.18 & 1.21 & 0.96 & 0.05 & 0.08 \\
\hline Authority & $0.54 * *$ & -0.22 & 1.14 & 0.98 & 0.04 & 0.08 \\
\hline Purity & $0.54 * *$ & -0.04 & 1.11 & 0.99 & 0.04 & 0.06 \\
\hline
\end{tabular}

Note: Path A corresponds to the path between condition and guilt, and Path B corresponds to the path between guilt and intuition as indicated in Figure 1. Full diagrams and results of the structural equation models and its results are available upon request from the first author.

$* p<0.05$ (one-tailed); $* * p<0.05$ (two-tailed). 


\section{Limitations}

The first limitation relates to a concern regarding the quality of our observations and the relatively low reliabilities found for some of the intuition salience measures. However, there is reason to believe that these lower reliabilities may actually increase confidence in patterns observed. While the reliabilities for the moral intuitions would be considered marginally low, there are several reasons why their influence on the tests of the hypotheses is minimal. First, the factor structure of the intuitions was confirmed prior to hypothesis testing. So, while the low reliability might indicate inconsistency in a measure, it does not indicate a lack of validity of the constructs' measurement. Second, low reliability always results in the statistical attenuation of relationships, ${ }^{16}$ and, as such, these lower reliabilities would have decreased our ability to observe the patterns found. Third, because the low reliability is limited to the final variable in our causal chain, it cannot affect assessments of the validity of our model. ${ }^{17}$ Only low reliability in a mediator variable can cause a model that should not fit to appear valid, or a model that should fit to appear invalid. In our study, the mediating variable has high reliability $(\alpha=0.88)$, and therefore any concern about the validity of our model cannot be attributed to low reliability. In fact, as stated above, the low reliabilities provide a more robust test of the processes hypothesized due to attenuation.

The second limitation relates to the fact that we examined only one game and the mediating influence of only one moral emotion. Questions remain about the manner in which different games and different moral emotions (both positive and negative) mediate the relationship between game play and the salience of all moral intuitions. For example, we examined the negatively valenced moral emotion of guilt. Other moral emotions, especially positive moral emotions such as pride, may have similar or dissimilar influences on moral intuitions, both in terms of the direction and intuition specificity. The findings here indicate that negatively valenced moral emotions elicit positive, intuition-specific changes. It is unclear whether this is true for positive moral emotions, such as pride. Based on the moral self-licensing literature, which shows that being good can give individuals a "license" to misbehave, ${ }^{18}$ it is plausible to suspect that positive moral emotions would have a negative influence. Therefore, it remains unclear whether guilt is a unique moral emotion, whether it behaves similarly to other negative moral emotions, and whether positive moral emotions have similar effects.

Finally, we would note the lack of covariates utilized in the current study. Numerous variables, including gender, game play experience, and political affiliation, may be expected to interact with guilt or moral intuition salience. We examined this potential in our data analyses, and all results were robust to the inclusion of these covariates. Still, future research should examine the potential for individual differences to moderate the results presented here.

\section{Conclusion}

Prior research has argued that antisocial video games may yield prosocial outcomes. ${ }^{1,2,19}$ The current paper tests this possibility and provides evidence that committing immoral behaviors in a video game can lead to an increased moral intuition salience. Contrary to popular belief, engaging in heinous behaviors in virtual environments can lead to an increased sensitivity to moral issues. Whether this heightened sensitivity should then translate to sterner moral judgments and a stronger sense of morality for the player remains to be determined. Research should continue to explore these issues, replicating the current research to determine whether these changes in moral sensitivity extend to real world increases in moral behavior, and whether similar effects are found with other moral emotions, such as pride.

\section{Notes}

a. Our decision to elicit guilt using a memory recall as a comparison to video game play rather than using other more traditional forms of media, such as television viewing, was related to the fact that guilt is an "emotion of self-assessment",20 as compared to emotions of "other assessment," such as contempt, anger, and disgust. ${ }^{21}$ One feels guilt when one commits a moral violation, whereas contempt, anger, and disgust are felt when observing others' moral violations. As such, we did not feel that watching a media experience would be capable of eliciting guilt, as a viewer of a traditional media experience simply observes the actions of characters on screen; s/he does not actually engage in the immoral/moral behavior. As such, it is unlikely that viewing traditional media would lead to feelings of guilt. However, when one plays a video game character, one is actually engaging in simulated moral/ immoral behavior. This type of simulated experience should be capable of eliciting guilt. We thus compared guilt elicited from virtual experiences to guilt elicited from prior real life experiences through the utilization of a memory recall procedure.

\section{Author Disclosure Statement}

No competing financial interests exist.

\section{References}

1. Weaver AJ, Lewis NL. Mirrored morality: an exploration of moral choice in video games. Cyberpsychology, Behavior, \& Social Networking 2012; 15: 610-614.

2. Hartmann T, Toz E, Brandon M. Just a game? Unjustified virtual violence produces guilt in empathetic players. Media Psychology 2010; 13:339-363.

3. Tangney JP, Stuewig J, Mashek DJ. Moral emotions and moral behavior. Annual Review of Psychology 2007; 58: 345-372.

4. Tamborini R. (2012) A model of intuitive morality and exemplars. In Tamborini R, ed. Media and the moral mind. New York: Routledge, pp. 43-74.

5. Tamborini R. Moral intuition and media entertainment. Journal of Media Psychology 2011; 23:39-45.

6. Graham J, Haidt J, Koleva S, et al. Moral foundations theory: the pragmatic validity of moral pluralism. Advances in Experimental Social Psychology 2013; 47:55-130.

7. Joeckel S, Bowman N, Dogruel L. Gut or game? The influence of moral intuitions on decisions in video games. Media Psychology 2012; 15:460-485.

8. Lewis RJ, Grizzard M, Bowman ND, et al. (2011) Intuitive morality and reactions to news events: responding to news of the Lockerbie bomber's release. Annual Meeting of the Broadcast Education Association, Las Vegas, Nevada.

9. Tamborini R, Prabhu S, Lewis RJ, et al. (2012) Media's influence on the salience of moral intuitions and egoistic motivations. Annual Conference of the National Communication Association, Orlando, FL. 
10. Graham J, Haidt J, Nosek BA. Liberals and conservatives rely on different sets of moral foundations. Journal of Personality \& Social Psychology 2009; 96:1029-1046.

11. Napier JL, Luguri JB. Moral mind-sets: Abstract thinking increases a preference for "individualizing" over "binding" moral foundations. Social Psychological \& Personality Science 2013; 4:754-759.

12. Grewal R, Cote JA, Baumgartner H. Multicollinearity and measurement error in structural equation models: Implications for theory testing. Marketing Science 2004; 23: 519-529.

13. Hu L, Bentler PM. Cutoff criteria for fit indexes in covariance structure analysis: conventional criteria versus new alternatives. Structural Equation Modeling: Multidisciplinary Journal 1999; 6:1-55.

14. Gentile D. Pathological video-game use among youth ages 8 to 18: a national study. Psychological Science 2009; 20:594-602.

15. Heuer F, Reisberg D. Vivid memories of emotional events: the accuracy of remembered minutiae. Memory \& Cognition 1990; 18:496-506.

16. Hunter JE, Schmidt FL. (1994) Correcting for sources of artificial variation across studies. In Cooper H, Hedge LV, eds. The handbook of research synthesis, New York: Russell Sage Foundation, pp. 323-336.
17. Hunter JE, Gerbing, DW. Unidimensional measurement, second order factor analysis, and causal models. Research in Organizational Behavior 1982; 4:267-320.

18. Merrit AC, Effron DA, Monin B. Moral self-licensing: when being good frees us to be bad. Social \& Personality Psychology Compass 2010; 4/5:344-357.

19. Ferguson CJ. Blazing angels or resident evil? Can violent video games be a force for good? Review of General Psychology 2010; 14:68-81.

20. Taylor G. Pride, shame, and guilt: emotions of self-assessment. New York: Oxford University Press.

21. Rozin P, Lowery L, Imada S, et al. The CAD triad hypothesis: a mapping between three moral emotions (contempt, anger, disgust) and three moral codes (community, autonomy, divinity). Journal of Personality \& Social Psychology 1999; 76:574-586.

Address correspondence to: Dr. Matthew Grizzard 359 Baldy Hall University at Buffalo The State University of New York Buffalo, NY 14260

E-mail: mngrizza@buffalo.edu 